\title{
Belphégor
}

\section{Le récit de vie de vedette, L'invention d'un genre : Rigolboche, Thérésa, Paulus}

\section{Marie-Ève Thérenty}

\section{(2) OpenEdition}

1 Journals

Édition électronique

URL : https://journals.openedition.org/belphegor/279

DOI : 10.4000/belphegor.279

ISSN : 1499-7185

Éditeur

LPCM

Référence électronique

Marie-Ėve Thérenty, «Le récit de vie de vedette, L'invention d'un genre : Rigolboche, Thérésa, Paulus », Belphégor [En ligne], 11-1 | 2013, mis en ligne le 07 juillet 2013, consulté le 07 décembre 2022. URL http://journals.openedition.org/belphegor/279; DOI : https://doi.org/10.4000/belphegor.279

Ce document a été généré automatiquement le 29 septembre 2020.

\section{(c) (i) (9)}

Creative Commons - Attribution - Pas d'Utilisation Commerciale - Pas de Modification 4.0 International - CC BY-NC-ND 4.0

https://creativecommons.org/licenses/by-nc-nd/4.0/ 


\title{
Le récit de vie de vedette, L'invention d'un genre : Rigolboche, Thérésa, Paulus
}

\author{
Marie-Ève Thérenty
}

1 Les années 1850-1860 voient fleurir la littérature biographique qui répond à un horizon d'attente, à une pratique déjà bien codifiée ${ }^{1}$, en pleine expansion et qui trouve un relais dans la curiosité croissante du public pour les figures célèbres de la vie intellectuelle ou artistique. L'exemple le plus connu de cette mode est la série de biographies publiées par Eugène de Mirecourt sur ses contemporains. Il s'agit de petites plaquettes sommaires qui compilent des renseignements biographiques, des anecdotes croustillantes, avec un portrait et un autographe. Il en fit même, à partir de 1857, un journal biographique, Les Contemporains. À côté des petits journaux qui exploitent cette veine, figurent aussi des mémoires de grands écrivains diffusés avec beaucoup de publicité dans la presse, comme ceux d'Alexandre Dumas publiés entre 1852 et 1856 . Un lien se forme visiblement entre le biographique et le médiatique. Mais cette folie biographique s'empare aussi de la vie artistique comme le prouvent les mémoires de mademoiselle Mars² publiés en 1849 et ceux de Mademoiselle Georges rédigés en $1857^{3}$. Rien d'étonnant alors à ce qu'en plein essor de la mode du cancan et du café-concert, apparaissent les premiers mémoires de danseuses et de chanteuses. Pour explorer l'invention de ce genre de l'« autobiographie » de vedette, nous avons sélectionné trois ouvrages : les mémoires de Rigolboche, la danseuse de cancan, parus en 1860, ceux de Thérésa, la chanteuse de café-concert en 1865 et enfin publiés beaucoup plus tardivement les souvenirs de Paulus, le chanteur, en $1908^{4}$. Ce corpus hétérogène (deux femmes/un homme; deux chanteurs/une danseuse; des mémoires de fin de vie /des souvenirs de jeunesse) est intéressant par les traits communs qu'il met en valeur : à travers ces trois exemples de mémoires se fixe un premier stade du genre, beaucoup plus médiatique qu'intime.

2 Reprenons les différents contextes de ces publications. Les mémoires de Rigolboche, danseuse de cancan à la mode, lancée par quelques journalistes de petits journaux, 
connaissent un gros succès de scandale en 1860. Se seraient écoulés rapidement 60000 exemplaires de l'ouvrage. Dans les semaines qui suivent la sortie, toute une littérature dérivée voit le jour : Rigolchianna, Album comique contenant les réflexions de M. Flamèche au sujet des mémoires de madame Marguerite Rigolboche; A bas Rigolboche! sans portrait, $n i$ vignette; La Rigolbochomanie, croquis lithographiques et chorégraphiques ${ }^{5}$. Une polémique se développe dans les journaux: doit-on accepter que Rigolboche incarne le temps présent ? Thérésa, la chanteuse de café-concert, se saisit du filon en 1865 en publiant à son tour ses mémoires. Elle vend 75000 exemplaires de ses souvenirs en deux ans et autorise même Morel en 1868 à en écrire la suite, moins connue, qui paraît sous le titre de Nouveaux mémoires de Thérésa ${ }^{6}$. Là aussi de vives protestations se font jour ${ }^{7}$. On voit paraître des parodies, surgir des vaudevilles comme les Mémoires de cherchez-moi-ça ou les Mémoires de Réséda $a^{8}$. Le dernier ouvrage écrit une quarantaine d'années plus tard est sensiblement différent : il s'agit d'un ouvrage de fin de vie où Paulus, vedette un peu oubliée, aidé d'un chansonnier, Octave Pradels, fait à la fois l'histoire de sa carrière et celle du café-concert. L'ouvrage, soigné, abondamment illustré par des photographies et entrecoupé de chansons est publié par livraisons. Il ne connaît pas le succès de scandale de ses prédécesseurs. Ces trois textes dessinent donc une déclinaison intéressante qui permet de déterminer le prototype du récit de vie de vedette au XIX siècle. Il n'est pas évident d'ailleurs qu'il s'agisse d'autobiographie, ni même de biographie. Ces récits relèvent de catégories hybrides et sans doute afin de les comprendre faudra-t-il les replonger dans leur univers journalistique et mettre en valeur leur caractère de pur tourniquet médiatique. Ce sont des métatextes sur la réclame, sur le journalisme et même sur le récit de vie de vedette telle qu'il va se développer au XXe siècle.

\section{Des récits de vie exemplaires et prototypiques}

3 Concetta Condemi, l'historienne du café-concert, a sur la pratique des mémoires des mots sévères mais probablement justes : «Écrits à des fins commerciales par des tiers, pour un public friand de " contes de fées ", ces textes peu fiables retracent des parcours exemplaires pour inspirer au lecteur des rêves de mobilité sociale et de réussite ${ }^{9}$ ». Effectivement, si ces mémoires prétendent répondre à un appel du public et à une nécessité sociale, de fait, ils se conforment à une exigence médiatique, celle de la réclame, et ce faisant, ils contribuent à structurer un imaginaire collectif autour de la notion de vedette ou d'étoile.

\section{Structure du récit de vie}

4 La formule, qui est encore aujourd'hui celle des récits de vie médiatiques dans les magazines people, est déjà bien rodée dès les Mémoires de Thérésa. Le récit de vie ne suit généralement pas le déroulement chronologique de la vie de la vedette comme dans une autobiographie classique mais commence par un portrait en gloire de l'artiste au sommet de son art. Ainsi les mémoires de Paulus débutent par une image arrêtée en 1886, au moment où il a créé la chanson "En revenant de la revue ». Il s'y montre comme favorisant l'ascension du général Boulanger en le popularisant. Le coup de force du chanteur, au seuil de son récit, est de se poser comme celui qui fait les puissants. L'analepse se fait ensuite en deux temps: Paulus relate d'abord ses débuts en 1868 à vingt-trois ans à l'Eldorado puis ensuite seulement il en arrive à raconter son enfance. 
Le suspense ne réside donc pas, comme dans un roman, dans le devenir (le devenir, tout le monde le connaît...) mais à la rigueur dans le passé : comment l'artiste en est-il arrivé là ? La narration commence donc à rebours.

Le temps de la petite enfance, même s'il n'est abordé que dans un deuxième temps, est décrit longuement, comme un biographème important. Il s'agit de montrer qu'enfant, l'artiste se distingue par des qualités peu communes. Thérésa, dès trois ans, fait son numéro de revue chez les voisins :

À l'âge de trois ans, je savais par cœur toutes les chansonnettes à la mode. Je les fredonnais soit dans la cour de la cité, soit dans l'escalier de la maison. Les voisins m'avaient prise en grande affection; on m'invitait à dîner dans toutes les mansardes du voisinage, et au dessert, la petite chanteuse - c'est ainsi qu'on m'appelait déjà - disait, de sa petite voix flûtée, les chansons qui couraient les rues $^{10}$.

Paulus, littéralement dès son premier cri, dévoile ses capacités vocales exceptionnelles :

Saint-Esprit, commune de Bayonne (Basses-Pyrénées), entendit mes premiers cris, lesquels furent si vigoureux que la vieille sage-femme béarnaise résuma son impression favorable par ces mots prononcés dans son patois: "Il en aura un galoubet, ce petit là !» La prédiction de la bonne femme s'est réalisée ; plus tard, un grand critique me surnomma : La trompette populaire ${ }^{11}$.

6 L'enfant se caractérise d'emblée par sa vocation et il ne fait que devenir, qu'accomplir ce qu'il est déjà en puissance. Le passé préfigure le présent même s'il est aussi un antiprésent (car la vedette vient des milieux les plus humbles).

7 Cette anticipation sur le destin explique que le récit soit miné par cette inversion chronologique. Il se condamne à une vaste redondance où chaque séquence ne fait que répéter le postulat de départ : le caractère exceptionnel hors norme du chanteur ou de la chanteuse. Le récit est donc principalement composé de noyaux répétitifs qui ont pour fonction de corroborer la thèse. Tout le reste est éludé comme le montre la manière dont Thérésa évite de raconter ses années sans chanson: "Comment se passèrent les quatre années qui me séparaient de l'âge où l'enfant devient jeune fille, c'est ce que je ne sais plus ${ }^{12}$ ». Ses séquences répétées peuvent même créer un effet monotone: la vie de Paulus paraît n'être rythmée que par ses engagements et ses succès dans différents cafés-concerts.

8 Ces répétitions se structurent selon deux schèmes récurrents et complémentaires : la convocation et l'épreuve. La convocation est la scène où une intervention fulgurante lance le héros sur la voie de l'accomplissement. Ainsi Thérésa met en scène le moment où par hasard elle tyrolianise le refrain d'une romance, trouvant sa veine de chanteuse comique.

9 J'eus personnellement un succès prodigieux.

On me fit bisser.

Je bissai en adoptant un accent allemand et en tyrolianisant le refrain.

Les applaudissements éclatèrent

Goubert demeura silencieux. ${ }^{13}$

10 Cette scène canonique où Thérésa a une révélation grâce à Goubert confirme la vedette dans son être. Paulus raconte ses débuts de gambillard exactement de la même manière. Mais l'autobiographie a également une dimension agonique : «J'engage une 
bataille vis-à-vis de moi-même ${ }^{14}$ écrit Rigolboche. Elle court alors d'épreuve en épreuve. Paulus raconte son échec à l'Eldorado en 1868. Il est au bout d'un mois chassé et part en province. Tel un Rastignac de la chansonnette, il raconte dramatiquement sa reconquête de la scène parisienne.

\section{Questions d'identité} de l'identité. L'autobiographe de vedette hésite entre l'affirmation de la permanence et la mise en avant de changements radicaux. D'un côté, pour le dire en chanson, » je n'ai pas changé ». Le chanteur ou la chanteuse populaire affirme invariablement son appartenance au peuple et met en place un raisonnement circulaire : j'appartiens au peuple, c'est pourquoi le peuple fait mon succès. Thérésa le formule ainsi : «Je suis une fille du peuple, et j'amuse le peuple. C'est ainsi que je trouve moyen de ne pas me séparer de ma famille ${ }^{15}$ ». En même temps, l'autobiographie met en scène dans des formules volontairement paratactiques des mutations essentielles: " Il y a trois ans j'étais une pauvre fille inconnue; aujourd'hui mon nom est célèbre ${ }^{16}$ ». L'autobiographie joue avec cette rengaine. Voici la même idée développée, selon le modèle du conte de fées :

(Le lecteur) a compris du premier coup que, pauvre fille jadis, sans ressources et sans avenir, je dois éprouver une joie immense de me voir aujourd'hui acclamée par la foule et fêtée par tout le monde, et que mes accès d'orgueil sont comme des remerciements à Dieu de m'avoir fait un présent doré après le présent sombre qu'il m'avait infligé ${ }^{17}$.

En gardant un équilibre entre le même et l'autre, il s'agit de raconter une ascension sociale hors du commun. Pour matérialiser cette réussite, la vedette détaille d'abord ses rencontres avec les grands de ce monde. Ainsi Thérésa écrit : «La première invitation de ce genre me fut adressée l'année dernière par un personnage qui porte un grand nom et qui me fit l'honneur insigne de me demander quelques chansons ${ }^{18} »$. La périphrase euphémistique cache mal Napoléon III d'autant que la rumeur publique, non confirmée et peu vraisemblable, a couru d'une liaison de l'empereur avec la chanteuse. Mais la vedette n'hésite pas à mesurer également sa réussite à une aune sonnante et trébuchante. La relation de la chanteuse à l'argent semble particulièrement décomplexée et ce point différencie la chanteuse de l'actrice par exemple. L'argent ne se cache pas et les chanteurs intègrent parfaitement la nouvelle donne mercantile de l'industrie du divertissement. «À la Scala commença l'ère de mes gros appointements ${ }^{19}$ » écrit Paulus. La comparaison avec le banquier et l'insertion dans le contexte de la révolution industrielle viennent naturellement à la bouche de Thérésa: «Le succès d'une de mes chansons nouvelles me préoccupe tout autant, sinon plus, que le succès d'une grosse opération financière n'inquiète les frères Péreire ${ }^{20} »$. Thérésa et Paulus sont des parvenus et affichent leur réussite financière : "Mon portefeuille regorgeait, ça ne pouvait pas durer. Je voulais avoir mon hôtel, mes gens, mes équipages et élever splendidement ma famille qui croissait toujours. Je dépensais 60000 francs par an. Me l'a-t-on assez reproché !'1 »

13 L'angoisse de la chute, de la faillite reste toujours perceptible dans ces textes. Rigolboche sait que sa gloire est éphémère ; quand il prend la plume, Paulus ne fait plus partie des grandes vedettes. Il décide d'ailleurs de ne pas raconter son déclin et de s'arrêter au sommet de sa gloire : «Le récit de ces dernières années serait d'un intérêt 
secondaire pour les lecteurs et je préfère m'arrêter à l'apogée de ma réussite, au sommet de la montagne gravie, sans conter la descente pénible sur l'autre versant ${ }^{22}$ ». La fin de l'autobiographie de Thérésa encore en plein succès pose l'angoissante question du lendemain.

Vous ne m'en voudrez donc pas, cher lecteur, de m'être préparée avec ce petit volume la consolation de voir mon nom survivre à mon succès, afin que le jour où j'aurai perdu ma voix, il me reste au moins mes mémoires ${ }^{23}$.

\section{Sommes-nous devant des vedettes?} encore vraiment fixée. «Je fais partie du bataillon des célébrités ${ }^{24}$ » dit Rigolboche Paulus et Thérésa préfèrent au terme de vedette celui d'étoile : «On voit déjà que l'on me traitait comme une étoile véritable ${ }^{25}$ » s'écrie par exemple Thérésa. Mais, même en faisant abstraction des questions de lexique, la réponse est à moduler suivant les cas. Rigolboche n'a pas le statut d'une vedette. En risquant l'anachronisme, elle constitue plutôt l'équivalent Second Empire d'une starlette éphémère à Cannes dans les années soixante ou d'un buzz sur le net aujourd'hui. Elle est construite par un système médiatique labile et inconstant qui découvre ses pouvoirs. Elle se décrit d'ailleurs ellemême comme un puff:

Ils se sont amusés à me rendre célèbre, tant pis pour eux, je suis leur égale maintenant.

J'exploite ma réputation ; que les prudes et les hommes graves s'en prennent à mes apologistes qui ont cru être très spirituels en me glorifiant ; à présent que c'est fait, je ne rentrerais pas dans l'obscurité pour un hôtel et des chevaux. ${ }^{26}$ comme l'a montré Dominique Kalifa ${ }^{27}$, entre 1860 et 1865 . Effectivement, entre Rigolboche et Thérésa, un changement de régime médiatique est visible. Entre temps, par exemple Le Petit Journal, premier journal à cinq sous, a été fondé et a même rapidement atteint des tirages quotidiens de plus de 300000 exemplaires. Lorsque Thérésa écrit 掼 « Or je suis aussi populaire que Timothée Trimm, Ponson du Terrail ou Jacques Offenbach $»^{28}$ 满, elle montre par ces références que sa popularité dont Le Petit Journal a été un des artisans, ne peut se comprendre que dans un système médiatique. Dans ses mémoires, la description précise de sa relation à son public fait surgir des phénomènes de fascination collective envers sa personne qui montrent bien la naissance du vedettariat avec le phénomène des fans.

Ils sont là cinq ou six cents, non dans l'intérieur du café-concert, mais au dehors, groupés autour des massifs de verdure qui nous servent d'enceinte. Haletants, avides de musique et de plaisir.

Leurs yeux sont fixés sur la chanteuse.

$\mathrm{Au}$ troisième couplet, ils savent tous les refrains de la chanson et les répètent en chœur ${ }^{29}$

pas à dire : j'étais devenu célèbre. Et la preuve, c'est qu'on me singeait. On imitait la coupe de mes habits : je faisais mon petit Prince de Galles. ${ }^{30}$ "

mane-t-il à Thérésa pour devenir une star comme les stars de cinéma dans les années vingt-trente? Peut-être cette aura érotique, cette fétichisation des corps. Thérésa met constamment en avant sa laideur et se présente comme parfaitement 
commune : «Qui ne sait d'ailleurs que toute femme, si peu jolie qu'elle soit, gravit à un moment donné ce que j'appellerai le calvaire de l'amour ${ }^{31}$ " Les stars de papier glacé décrites par Edgar Morin ${ }^{32}$ sont encore loin.

\section{La question du genre autobiographique}

Dans la partie précédente, le terme de récit de vie a été préféré à celui d'autobiographie car la question du genre paraît poser question comme le montrent d'emblée au seuil des ouvrages les trois titres. Thérésa et Rigolboche choisissent l'expression de Mémoires qui laisse attendre un rapport à l'intime et au social différent de celui de l'autobiographie. Mais dans les deux cas la notion de mémoires semble problématique, étant donné l'âge des protagonistes: en 1860, Rigolboche a dix-huit ans; en 1865, Thérésa 28. Les mémoires s'écrivent généralement en fin de parcours. Les soi-disant Mémoires de Rigolboche racontent en fait trois ou quatre ans de sa vie. Ce phénomène de retour précoce sur soi rapproche ces mémoires des récits biographiques médiatiques modernes comme le montre cette analyse des biographies parues dans Paris-Match: «Les vies que narre ce magazine appartiennent de préférence à ceux que distingue dans l'instant une promotion ou mieux encore la Promotion. Car le héros, selon Match est l'individu qui hier noyé dans la masse se retrouve soudain assis à la première place ${ }^{33}$ ». Ce choix mémorialiste laisse donc présupposer une intervention extérieure, un « teinturier » dirait-on dans le langage du dix-neuvième siècle, teinturier qui n'apparaît pas dans le paratexte des livres des deux femmes. En revanche, le titre de Paulus Trente ans de cafés-concerts, souvenirs recueillis par Octave Pradels encadre la vie du chanteur de manière totalement différente. Ici, la médiation accomplie par le chansonnier Octave Pradels n'est pas cachée mais exhibée. Le titre curieux semble inverser la polarité auctoriale faisant de Paulus un auteur et de Pradels le creuset des souvenirs. Ce lapsus dévoilant sans doute une part de vérité insiste sur la substitution des regards. Sur le monde des cafés-concerts qui constitue l'objet essentiel du livre, le regard est sans doute plutôt celui du chansonnier que du chanteur.

Malgré les indiscrétions, malgré la parole scandaleuse parfois, le je intime ne paraît pas être le centre de ces récits. Ils sont effectivement plutôt prétextes à donner un point de vue sur le monde des spectacles, sur les petits théâtres et les salles de café-concert ou à refaire circuler un certain nombre d'anecdotes éculées de petits journaux. Ainsi le livre de Rigolboche recueille la physiologie de la biche, des aphorismes graveleux, la description du Casino Cadet, celle des salons Markouski, du théâtre des délassementscomiques... Au fil des pages, le livre se transforme en un fourre-tout entre les «brèves de comptoir » et le recueil d'anas sur les blondes, les brunes, les rousses : " Toutes les jolies femmes sont brunes. Excepté celles qui sont blondes ${ }^{34} »$. Le sujet de l'autobiographie paraît peu à peu se réduire à une voix de régie moqueuse et parisienne. Le désir structurant et la volonté apologétique présents dans les mémoires d'artistes selon Sylvie Jouanny ${ }^{35}$ sont relativement faibles ici. Du côté de Paulus, les souvenirs sont constamment interrompus par des listes de noms propres et des notices biographiques de chanteurs du café-concert. Le projet double de Paulus est clairement explicité dès les premières pages :

C'est en me remémorant les souvenirs de ce 14 juillet que l'idée me vint de rassembler mes mémoires, d'y faire figurer les artistes de valeur que j'ai côtoyés, de reproduire les chansons à succès du Concert, avec le portrait de leurs auteurs et de leurs interprètes. 
Je parlai de ce projet à mon ami, Octave Pradels, qui fut un des chansonniers de la belle époque de l'Eldorado, et qui est très documenté sur le café-concert. Il accepta de me prêter son concours et nous nous mîmes à l'œuvre ${ }^{36}$. autobiographique. Pour justifier un Paulus omniscient sur tous les spectacles, le livre le met en scène, d'une manière peu crédible, chantant en province mais courant à Paris à chacun de ses moments libres pour s'instruire sur les cafés-concerts. Il constitue le foyer unique de perception alors que le livre regorge bien plus probablement de choses vues de Pradels. Le témoignage de Thérésa paraît tout aussi improbable à partir du chapitre sur le café du cirque car elle délimite très clairement les espaces du café selon des critères de genre : «Le rez-de-chaussée était réservé aux petites dames. Le premier étage aux artistes, auteurs, journalistes et directeurs. Le second étage aux amateurs de la bouillote ${ }^{37}$ ». Les mémoires ensuite restent longuement au premier étage dévoilant que, silencieusement, un relais de la focalisation s'est opéré. apologétique de ses mémoires l'empêche d'évoquer sinon par allusions sa vie de séducteur. On peut parler de mensonge par omission. Mais chez les deux femmes, c'est l'hybridation fictionnelle, caractéristique des écritures journalistiques de l'époque, qui affaiblit le pacte de vérité. La menace du factice ou de la fiction pèse d'emblée sur les Mémoires de Rigolboche lorsqu'ils esquissent quelques topoï de l'enfance de la jeune fille perdue : la mère marâtre qui bat l'enfant, le séducteur qui la déshonore. On se croirait dans Les Mystères de Paris. La position de Thérésa est encore plus ambiguë. La question de la vie amoureuse engendre, ce qui est rare dans ce texte, la déclinaison d'une forme de pacte de sincérité : «Ici j'éprouve un certain embarras. J'ai promis au lecteur d'être franche et de tout dire, et ce que j'ai maintenant à raconter touche au côté le plus délicat de ma vie. Je veux parler de mes amours ${ }^{38}$ ». Ensuite, l'histoire racontée, gorgée de clichés, relève de la mauvaise romance. Elle réécrit l'histoire de Manon et Des Grieux, le parodique étant d'autant plus visible qu'elle se retrouve avec son amant au Havre, lieu crucial de l'intertexte. De même la mort du père est l'occasion d'une véritable scène de feuilleton avec des effets de suspense mélodramatique grâce à des microchapitres qui miment la coupe feuilletonesque. L'effet parodique est patent et ne saurait être imputé à la chanteuse. Les commentateurs ont beau jeu de dénoncer la mise en scène et la fiction fabriquée par des tiers.

A la vérité, je me méfiais. Les auteurs des Mémoires de Thérésa, par elle-même, parus en 1865, sont, je le sais, de deux hommes d'esprit, Albert Wolff et Ernest Blum qui ont brodé sur les renseignements volontairement vagues dont ils ont farci et assaisonné l'autobiographie supposée. ${ }^{39}$

Effectivement dans les trois cas, l'autobiographie est rédigée à plusieurs mains. Si la collaboration précise autour de ces mémoires est parfois difficile à démêler 㡠 plusieurs versions circulent 㡠, un personnage paraît cependant central dans la production d'au moins deux de ces textes: le journaliste républicain et vaudevilliste Ernest Blum (1836-1907). Ernest Blum, ancien petit journaliste au Charivari, est impliqué seul ou avec Louis Huart, journaliste et directeur de théâtre dans la confection des Mémoires de Rigolboche. On cite aussi parfois Alexandre Flan, un jeune vaudevilliste, ce qui mettrait les Mémoires de Rigolboche encore plus radicalement du côté de l'ouvrage de blague et de café. Il n'est même pas sûr que le sujet ait participé à l'élaboration de sa propre autobiographie : «Elle resta complètement étrangère à la rédaction de ce petit volume, publié sous son nom et dont les procréateurs furent MM. Blum et Huart ${ }^{40} »$. 
24 Ernest Blum réitère la bonne opération avec Thérésa. Cette fois il s'offre la collaboration d'Albert Wolff, chroniqueur au Figaro et peut-être, si l'on en croit certaines versions, celle d'Henri Rochefort pour le dernier chapitre. Henri Rochefort revendiquera en tout cas la paternité des Mémoires. Nous disposons sur la fabrique de ce recueil d'un témoignage rétrospectif tardif mais de première main, celui d'Albert Wolff :

Un beau matin parurent les Mémoires de Thérésa. La diva de la chanson prenait la parole pour raconter au public sa vie tout entière en un volume à trois francs cinquante dont quarante mille exemplaires furent vendus en huit jours. Deux ans auparavant, mon vieux camarade Ernest Blum, poussé par les besoins d'un début laborieux et ayant de nombreuses charges, avait fait un coup de maître : avec un petit bouquin à couverture rose, contenant, sous le titre alléchant de Mémoires de Rigolboche, quelques potins agréablement tournés sur les coulisses des Délassements-comiques, Ernest Blum avait gagné une vingtaine de mille francs. Comment se fit ce livre? C'est une épopée! Thérésa qui est une artiste classée aujourd'hui, ne m'en voudra pas si j'entre dans la série des révélations. Elle nous donna quelques indications sur son origine que nous entourâmes de pas mal de poésie. Moi personnellement $\mathrm{j}$ 'inventai pour la circonstance un père fantaisiste à Thérésa, un vieux ménétrier qui, après le labeur du jour, enseignait la musique à sa petite Thérésa. Pas un mot de vrai dans cette légende, qui, néanmoins à la lecture impressionna si vivement la diva populaire, qu'elle versa d'abondantes larmes sur ce souvenir de son enfance ${ }^{41}$.

Les mémoires sont d'ailleurs déclinés en vaudeville sous le titre de Mémoires de réséda, souvenirs contemporains donné en 1865 au théâtre du Palais-Royal. Cette pochade dure plusieurs mois. Les auteurs Wolff, Rochefort et Blum y assument cette fois leur véritable identité et responsabilité auctoriale.

Malgré l'intervention d'un tiers, il est difficile de parler de biographie, l'énonciation et la simulation autobiographique tout comme la dérive médiatique invitant à voir ici un genre à part entière, la supercherie médiatique, dont les mémoires de Rigolboche et ceux de Thérésa constituent une forme de prototype. Même si l'auteur des souvenirs de Paulus n'est pas un journaliste, Trente ans de café-concert se conforme dans une certaine mesure à ce modèle structurant.

\section{Des supercheries médiatiques}

Une phrase de Rigolboche 罂 « Voilà ce que c'est que de jouer avec maman la réclame ${ }^{42}$ ” F⿱ ${ }_{20}$ pourrait figurer en épigraphe de cette partie. Si les mémoires de Rigolboche et ceux de Thérésa peuvent se prêter à une lecture au premier degré, ils peuvent également engendrer un vrai plaisir ludique dû à la mystification qui se joue à plusieurs degrés dans ces œuvres et qui fait entrer le lecteur dans un tourniquet médiatique.

Autant qu'une mise en valeur des artistes, ces mémoires sont une façon de valoriser leurs auteurs par la private joke comprise seulement des initiés. Le texte fourmille donc d'allusions cryptées à ses vrais auteurs qui passent, tel Hitchcock dans ses films, comme des silhouettes d'arrière-plan. Ainsi Rigolboche parle d'Ernest Blum, promettant dans un amusant retournement de ne pas donner d'indications sur sa vie privée :

Je dirais bien quelques mots de sa personne, mais il est si modeste que je craindrais de le blesser. N'est-ce-pas, Ernest Blum, que cela te serait désagréable que je parlasse de toi?

Rassure-toi, mon fils, je ne dirai rien; je ne te ferai pas le vilain tour de rien 
raconter de tes amours ni de tes affaires, tu es trop gentil et surtout... trop journaliste ${ }^{43}$. hommage aux journalistes qui les ont faites. L'ensemble du dix-huitième chapitre des mémoires de Thérésa est consacré à la presse. Le premier journaliste qu'elle félicite et remercie est bien sûr Albert Wolff non pour la rédaction des mémoires mais pour l'avoir soutenue dans Le Nain jaune. Un peu plus loin, Thérésa fait un long panégyrique du Figaro et de son directeur Villemessant, employeur de Wolff. La chanteuse sert les hommes de presse qui servent la chanteuse : le tourniquet médiatique est en marche. «On vous connaît parce que j'existe ${ }^{44}$ » dit Rigolboche à Mané, formulant un paradoxisme assez juste.

31 A contrario, elles s'en prennent aux journalistes qui les ont attaquées. Le livre est le moyen pour Rigolboche de créer une scie contre Jules Janin. «L'autre jour, (ma tante) voulait aller arracher les yeux à $\mathrm{M}$. Jules Janin parce qu'il avait latinisé sur mon coup de pied $^{45}$ ». Janin est moqué à plusieurs reprises dans le livre, selon une stratégie du ricochet. La danseuse se venge de son mépris sans vraiment que ni elle ni les auteurs ne risquent représailles car Janin ne peut, dans un système médiatique, que savoir gré aux petits journalistes de le citer dans un ouvrage à succès. Parfois, il est vrai, des comptes politiques se règlent dans le ridicule jeté sur tel ou tel. De Louis Veuillot, Rigolboche écrit : « Il élève la voix, je lève la jambe ; nous sommes faits pour nous comprendre ${ }^{46}$ ».

Mais la circulation des noms et des textes, inaugurée ici excède l'espace des mémoires puisque par un phénomène de retour, les journaux de l'époque citent extensivement les mémoires de Thérésa ou de Rigolboche. Et en 1913, au moment de la mort de Thérésa, les articles nécrologiques reprendront encore ce puff des mémoires en citant avec ou sans guillemets. Le jeu citationnel est sans fin.

\section{Du style de journaliste plus que de vedette}

Cette énonciation cachée pose évidemment le problème du style des mémoires. Albert Wolff en donne, quelques années plus tard, la recette :

Huit jours après, parurent ces Mémoires extraordinaires où le public payait trois francs de plaisir de relire nos articles qu'il avait déjà lus pour trois sous dans nos journaux, et que nous avions adroitement intercalés entre cinq ou six chapitres complètement inédits ${ }^{47}$.

34

Effectivement, les mémoires de Rigolboche et de Thérésa se composent d'une collection d'aphorismes, de nouvelles à la main et de petits articles. Ainsi, au mépris de toute vraisemblance s'intercalent dans la narration, on l'a vu, de petites saynètes de mœurs qui ne proviennent visiblement pas des artistes. Parfois un métadiscours justificatif tente d'expliquer la mosaïque en faisant de la chanteuse une plaque de réfraction de l'esprit du temps, un corps médiatique à elle toute seule en quelque sorte.

Une fois pour toutes, il faut que je prévienne le lecteur qu'il m'arrivera souvent, à chaque pas que je ferai dans mon récit, de raconter ce que j'aurai vu et ce qu'on m'aura dit, et de répéter au public des anecdotes qui l'intéresseront, comme elles m'ont intéressée moi-même. ${ }^{48}$ 
Très clairement, les mémoires de Thérésa accumulent les stylèmes du Petit Journal et du Figaro. Dès les premières pages, Thérésa qualifie ses mémoires de causerie, faisant ainsi explicitement référence à la chronique de petit journal. La mise en paragraphe correspond aussi à celle de la petite presse : des paragraphes très courts le plus souvent réduits à une page, une typographie aérée où le blanc mange l'espace se retrouvent dans les trois mémoires. Quand Trimm réceptionne dans une chronique les mémoires de Thérésa, il termine ainsi son article: "Le livre de Thérésa, qui ne choque ni les règles de la grammaire, ni la bienséance sera bien accueilli. /Il aura du succès comme ses chansonnettes. /Le lecteur applaudira comme l'auditeur. /Ce sera... comme si elle chantait $\aleph^{49}$. Effectivement, Thérésa, comme Paulus, entrelace son récit de références à ses chansons. Ce qui apparaît ici est le continuum textuel entre chanson, mémoire et texte journalistique en 1865. Le lien entre Thérésa et la petite presse n'est donc pas purement stratégique : il est beaucoup plus essentiellement lié au partage et à la mise en commun d'un savoir poétique et textuel. Ce savoir s'est constitué dans la confection de textes modernes entre oralité et presse, d'une littérature médiatique et populaire qui fusionne deux héritages, celui de la chanson populaire et celui de la chronique de quotidien.

\section{Du parodique}

Mais loin d'être seulement mimétiques, ces mémoires sont aussi très fortement parodiques et réflexifs. Ils constituent malgré leur relative platitude, ce qui est plus moins vrai d'ailleurs selon les textes, de la littérature au second degré. Chacun de ces succès modélise le genre et constitue un intertexte auquel se référer.

37 Les mémoires de Rigolboche et de Thérésa parodient à la fois les autobiographies en général, multipliant les lieux communs (l'enfance, le premier amour) mais aussi plus subtilement, ils se moquent de leur propre supercherie en la soulignant par des mises en abyme. Les mémoires de Rigolboche, ainsi, abondent en histoires de nègres littéraires ou de détournements de textes. Elle dénonce par exemple ceux qui copient leurs lettres d'amour dans des recueils ou qui les tirent de vaudevilles.

Et quand on pense que cette lettre est copiée textuellement dans un drame de M.

D’Ennery!

Et il y a des gens qui sifflent quelquefois ses pièces.

Les ingrats ! $!^{50}$

Pourtant, Rigolboche multipliant ce genre d'anecdotes ne fait jamais la relation entre son récit et les entreprises qu'elle décrit. Au lecteur de faire le rapprochement, de constater que cette œuvre qui se moque de tant de détournements textuels n'est, ellemême, qu'un énorme rapt et de s'en amuser au second degré. Ces mémoires dénoncent aussi par mise en abyme le fonctionnement pervers (ou vertueux) de la réclame : peu importe qu'on dise de vous du mal ou du bien, ce qui compte c'est qu'on parle de vous. Ainsi le livre-réclame de Rigolboche met en scène des personnages-réclames comme ce Markouski.

Il adore les journalistes et se couperait un doigt plutôt que de les mécontenter.

Il les inonde d'invitations, de prévenances et de soins.

Dans le but très habile de se faire faire des articles élogieux.

C'est un chasseur de réclames. 
Et cela lui réussit.

On parle presque autant de lui que de moi ${ }^{51}$.

La pirouette finale est révélatrice. Les mémoires de Rigolboche décryptent à la fois son propre fonctionnement de mémoires scandaleux mais également anticipent toute une production d'autobiographies de vedettes dont ils démontent les rouages. Il est ensuite difficile de se positionner par rapport à un métatexte si lucide sur sa propre vanité. Thérésa est contrainte à un positionnement de contorsionniste pour créer un écart avec ce modèle qui s'impose dès les premières pages :

Un soir de l'année dernière, j'avais chanté le chemin du Moulin et le public, avec sa bienveillance habituelle, me rappelait et me redemandait le dernier couplet de la chanson que j'avais mise à la mode. Fiorentino qui m'avait fait l'honneur de venir m'entendre, s'écria

- C'est la Rigolboche de la chansonnette !52

Thérésa dégage alors une hiérarchie entre Rigolboche et elle, entre l'état éphémère de danseuse de cancan et son statut de chanteuse à voix. « " Et si j'hésite encore à écrire mes Mémoires, c'est que Rigolboche a écrit les siens ॥ $^{53}$. Même en reprenant les recettes de l'atelier mémorialiste de la danseuse, elle tente de mettre ces mémoires à distance pour mieux occuper une place de précurseur. C'est sans doute pour cette raison que le titre comporte cette mention redondante: «mémoires de Thérésa écrits par ellemême ».

Intéressant est le positionnement de Paulus par rapport à cette généalogie. Il fait référence à Rigolboche mais ce sont surtout les mémoires de Thérésa qui fonctionnent comme modèle, sans doute en raison de la stature de chanteuse. L'attitude de Paulus par rapport à ce texte matriciel montre qu'il a intégré un certain processus de construction de l'autobiographie à partir de réinvestissements de textes usés, de plagiat et de puff. Il cite un long passage des Mémoires de Thérésa sans guillemets pour raconter les débuts de la chanteuse, extrait qu'il insère dans le chapitre de ses propres débuts $^{54}$. Il s'agit d'un hommage mais cette citation masquée montre que le lecteur a encore affaire à la même génération de textes fondée sur le bricolage hétéroclite.

Beaucoup de traits caractéristiques de ce genre abondamment pratiqué que sera l'autobiographie de vedette sont déjà présents chez ces trois précurseurs : la nécessité de recourir à un professionnel de l'écriture pour la mise en forme des mémoires, la recherche du scandale et surtout la prise de conscience que la vedette est un phénomène purement médiatique. Mais plusieurs traits empêchent encore le genre de s'accomplir parfaitement. Les autobiographes n'ont pas conscience qu'il convient de véritablement entrer dans la conscience de la vedette, dans son intimité. Les mémoires de cette époque jouent donc sur tous les tableaux, veulent informer (Paulus), amuser (Thérésa) ou scandaliser (Rigolboche). Les mémoires sont bridés par tout un éboulis de textes qui se déverse là et surtout par le fait que la plume, extérieure au sujet, ne s'oublie jamais. Toute cette hétérogénéité opacifie les mémoires et empêche d'avoir accès à la voix authentique du chanteur ou de la chanteuse. Mais à la place surgit, notamment dans les mémoires de Rigolboche et de Thérésa, une voix métatextuelle qui dénonce et déconstruit, avant même que le genre soit pleinement inventé, un de ses travers pérennes : le jeu pervers avec la réclame. 


\section{NOTES}

1. Voir la mode des mémoires apocryphes des années 1820 .

2. Mémoires de mademoiselle Mars de la comédie Française, publiés par Roger de Beauvoir, chez Gabriel Roux et Cassanet éditeurs, 1849.

3. Et publiés en 1908.

4. Mémoires de Rigolboche, Paris, chez tous les libraires, 1860 ; Mémoires de Thérésa écrits par ellemême, Dentu, 1865 ; Paulus, trente ans de café-concert, souvenirs recueillis par Octave Pradels, Paris, société d'édition et de publications, 1908.

5. Alexis Dalès, Rigolchianna, Album comique contenant les réflexions de M. Flamèche au sujet des mémoires de madame Marguerite Rigolboche.; Stanislas de Charnal et E. Moreau de Bavière, À bas Rigolboche: sans portrait ni vignette, Paris, chez tous les libraires, 1860 ; Charles Vernier, La Rigolbochomanie, croquis lithographiques et chorégraphiques, Le Charivari, 1860.

6. Henry Morel, Nouveaux mémoires de Thérésa, A. Faure, 1868.

7. Voir par exemple Le Passe-temps, Journal de l'entracte, 5 et 12 mars 1865, Le Trou du souffleur, 30 mars 1865, "Assez de mémoires comme ça ", Le Tintamarre, dimanche 26 mars 1865 ; le Journal amusant, 4 mars 1865 .

8. Rochefort, Blum et Wolf, Les mémoires de Réséda, souvenirs contemporains, Paris, E. Dentu, 1865 (Paris, Palais-Royal, 4 mai 1865).

9. Concetta Condemi, Les cafés-concerts, histoire d'un divertissement, 1849-1914, Paris, Edima, 1992, p. 140.

10. Thérésa, op. cit., p. 10.

11. Paulus, op. cit., p. 61.

12. Thérésa, op. cit., p. 48.

13. Thérésa, op. cit., p. 220.

14. Rigolboche, op. cit., p. 72.

15. Thérésa, p. 2.

16. Thérésa, op. cit., p. 300.

17. Thérésa, op. cit., p. 254.

18. Ibid, p. 310.

19. Paulus, p. 172

20. Thérésa , p. 316.

21. Paulus, p. 323.

22. Ibid, p. 435.

23. Thérésa, p. 316.

24. Rigolboche, p. 6.

25. Thérésa, p. 253.

26. Rigolboche, p. 6.

27. Dominique Kalifa, La Culture de masse en France, La découverte, 2001.

28. Thérésa, p. 3.

29. Thérésa, p. 234.

30. Paulus, p. 341.

31. Thérésa, p. 156

32. Edgar Morin, Les Stars, coll. Points, Seuil, 1972.

33. Dubois Jacques, Edeline Francis, Klinkenberg Jean-Marie, Minguet Philippe, Pire François, Trinon Hadelin. «Les biographies de Paris Match». In: Communications, 16, 1970. Recherches rhétoriques. p. 110.

34. Rigolboche, p. 174. 
35. Sylvie Jouanny, L'actrice et ses doubles. Figures et représentations de la femme de spectacle à la fin du XIXe siècle, Droz, 2002.

36. Paulus, p. 15-16.

37. Thérésa, p. 57.

38. Thérésa, p. 156.

39. «Un pèlerinage au berceau de Thérésa », JNL, 6 octobre 1936.

40. Frédéric Loliée, La Fête impériale, Jules Tallandier, 1926, p. 229.

41. Albert Wolff, La Gloire à Paris, Victor Havard, 1886, p. 185.

42. Rigolboche, p. 6.

43. Rigolboche, p. 127.

44. Rigolboche, p. 2.

45. Ibid., p. 15.

46. Ibid., p. 8.

47. Albert Wolff, ibid.

48. Thérésa, p. 26.

49. Le Petit Journal, 17 février 1865.

50. Rigolboche, p. 59.

51. Rigolboche, p. 93.

52. Thérésa, p. 1.

53. Thérésa, p. 6.

54. Paulus, chapitre II.

\section{RÉSUMÉS}

Cet article porte sur la naissance d'un genre, le récit de vie de vedette, à partir de trois exemples, les mémoires de Rigolboche (1860), Thérésa (1865) et Paulus (1908). Il étudie la construction de ces récits et montre comment ils contribuent à élaborer un imaginaire collectif autour de la notion naissante de vedette. Rédigé à plusieurs mains, le récit de vie de vedette est une autobiographie dévoyée où la vérité et l'intimité ne sont pas des valeurs cruciales. Ces récits de vedette, du fait de leur dimension métatextuelle dévoilent, avant même que le genre ne soit pleinement activé, tous ses travers: l'obsession publicitaire, la recherche du scandale, la dimension mystificatrice...

\section{INDEX}

Mots-clés : chanson, biographie, café-théâtre, petite presse, réclame, vedettariat, culture médiatique, cancan 


\section{AUTEUR}

\section{MARIE-ÈVE THÉRENTY}

Rirra 21-Université de Montpellier III- IUF

Marie-Eve Thérenty est professeur de littérature française à l'université de Montpellier III, directrice du centre de recherches RIRRA 21 (littératures française et comparée, cinéma, musique, théâtre, arts plastiques), et coresponsable avec Guillaume Pinson du site et du projet medias19.org. Elle est spécialiste des rapports entre presse et littérature, de poétique du support et des imaginaires des sociétés médiatiques. Elle a publié notamment La Littérature au quotidien, Poétiques journalistiques au XIX ${ }^{e}$ siècle, Seuil 2007, codirigé La Civilisation du journal. Histoire culturelle et littéraire de la presse française au XIXe siècle, nouveau monde 2011 et avec Elisabeth Pillet, Presse, chansons et culture orale au XIX ${ }^{e}$ siècle, nouveau monde, 2012. Elle prépare un ouvrage sur les femmes journalistes de Delphine de Girardin à Florence Aubenas. 